\title{
Clinical significance of positive Pneumocystis jirovecii polymerase chain reaction in non-human immunodeficiency virus immunocompromised patients in a real practice
}

Hea Yon Lee ${ }^{1}$, Hye Seon Kang ${ }^{1}$, Hwa Young Lee ${ }^{1}$, Chin Kook Rhee ${ }^{1}$, Sook Young Lee ${ }^{1}$, Seok Chan Kim Seung Joon $\mathrm{Kim}^{1}$, Yeon Joon Park², Young Kyoon Kim , and Ji Young Kang ${ }^{1}$

${ }^{1}$ Division of Pulmonary Medicine, Department of Internal Medicine, ${ }^{2}$ Department of Laboratory Medicine, College of Medicine, The Catholic University of Korea, Seoul, Korea

Received: October 14, 2015

Revised : April 4, 2016

Accepted: April 4, 2016

\section{Correspondence to}

Ji Young Kang, M.D.

Division of Allergy and Pulmonary Medicine, Department of Internal Medicine, College of Medicine, Seoul St. Mary's Hospital, The Catholic University of Korea, 222 Banpo-daero, Seocho-gu, Seoul 06591, Korea

Tel: +82-2-2258-6060

Fax: +82-2-599-3589

E-mail: rkdwldud@catholic.ac.kr
Background/Aims: Pneumocystis jirovecii polymerase chain reaction (PCR) can be helpful in diagnosing Pneumocystis pneumonia (PCP); however it has limitations. We evaluated the prevalence of positive P. jirovecii PCR from non-human immunodeficiency virus (HIV) immunocompromised patients and tried to determine the risk of PCP development.

Methods: Between May 2009 and September 2012, P. jirovecii PCR was performed in bronchoscopic specimens from 1,231 adult non-HIV immunocompromised patients suspected of respiratory infection. Only 169 patients (13.7\%) who were tested positive for $P$. jirovecii PCR were enrolled. Retrospective chart review was performed. PCP was defined in patients with positive P. jirovecii PCR who were treated for PCP based on the clinical decision.

Results: From ${ }_{169}$ P. jirovecii PCR-positive patients, 90 patients were in the PCP group (53.3\%) and 79 patients were in the non-PCP group (46.7\%). In the PCP group, $38 \%$ of patients expired or aggravated after therapy, whereas the majority of patients $(84 \%)$ in the non-PCP group recovered without treatment for PCP. Independent risk factors for PCP by binary logistic regression analysis were underlying conditions- hematological malignancies, solid tumors or solid organ transplantation, dyspnea, age $<60$ years, and albumin $<2.9 \mathrm{~g} / \mathrm{dL}$.

Conclusions: This study suggests that not all P. jirovecii PCR-positive patients need to be treated for PCP. Among P. jirovecii PCR-positive patients, those who are less than 60 years old, with hematological malignancies, solid tumors or solid organ transplantation, low albumin, and with symptoms of dyspnea, the possibility of PCP might be higher. Treatment should also be selected to these patients.

Keywords: Immunocompromised; Polymerase chain reaction; Pneumocystis; Pneumonia

\section{INTRODUCTION}

Pneumocystis pneumonia (PCP) is a well-known oppor- tunistic infection that affects patients infected with human immunodeficiency virus (HIV) [1]. PCP, the incidence of which continues to increase, also occurs in 
immunocompromised patients without HIV, such as those with hematological malignancies or solid tumors; those who have undergone organ transplantation; and those receiving immunosuppressants, including corticosteroids [2-4]. Pneumocystis jirovecii may be present in the respiratory tracts of patients without clinical pneumonia. P. jirovecii polymerase chain reaction (PCR) positivity could reflect PCR contamination, colonization, or transient carriage of a very small number of $P$. jirovecii organisms, which has been defined as Pneumocystis colonization [5].

In immunocompromised patients without HIV, PCP progresses rapidly and causes severe respiratory failure. However, it is often difficult to diagnose. Standard microscopic examinations of sputum or bronchial specimens for P. jirovecii organisms have low sensitivity due to the low bacterial burden, and although lung biopsy can be confirmative, it is quite invasive $[6,7]$. Moreover, the rate of colonization is high in these patients. A recent study documented Pneumocystis colonization in induced sputum from $16 \%$ of patients with various autoimmune diseases [8]. The molecular detection tool P.jirovecii PCR provides a higher degree of sensitivity than do standard methods and can be useful for the clinical diagnosis of PCP [9]. However, its use is limited by P.jirovecii colonization, resulting in lower specificity. The clinical value of $P$. jirovecii PCR in diagnosing PCP in patients with systemic diseases has not been defined clearly.

The aim of this study was to evaluate the prevalence of $P$. jirovecii PCR positivity in respiratory samples from immunocompromised patients without HIV who underwent bronchoscopy due to suspected respiratory infection. Furthermore, we attempted to determine the risk of PCP development in these patients by comparing the treatment group (patients diagnosed with PCP) with the non-treatment group (those with suspected colonization).

\section{METHODS}

We collected and analyzed the results of $P$. jirovecii PCR performed on bronchial washing fluid or bronchoalveolar lavage fluid (BALF) from adult immunocompromised patients without HIV who were suspected of having respiratory infection at a 1,200-bed tertiary university teaching hospital between May 2009 and September 2012. Medical records of the patients with positive $P$. jirovecii PCR results were reviewed retrospectively. The study was approved by the Institutional Review Board of Seoul St. Mary's Hospital, which permitted evaluation and publishing of information from the patients' records. The requirement for informed consent was waived because of the retrospective nature of the study.

Definite PCP is diagnosed in the presence of a compatible clinical presentation of PCP and a positive immunofluorescent test in BALF $[1,10]$. In this study, we defined the presence of PCP as P. jirovecii PCR positivity and treatment for PCP based on respiratory symptoms and the detection of new pulmonary infiltrates on chest radiography or computed tomography. Patients with PCP were treated with trimethoprim-sulfamethoxazole (TMP-SMX) for 21 days; treatment with other anti-infective agents was withheld, except for patients with coinfections. Co-infected patients were additionally treated with appropriate empirical antibiotics, voriconazole, amphotericin, or ganciclovir, according to the coinfection diagnosed. Adjuvant corticosteroid therapy was administered to patients with partial pressure of arterial oxygen $\left(\mathrm{PaO}_{2}\right)$ values $<60 \mathrm{mmHg}$, as in the recommended regimen [11]. Patients without PCP (non-PCP group) were defined as $P$. jirovecii PCR-positive patients who did not require treatment for PCP, suggesting colonization or subclinical infection, rather than PCP.

The baseline clinical data included demographic characteristics, presence of underlying disease, previous use of medications (steroids and other immunosuppressants), and chemotherapy in the previous month; bronchoscopy results, including those from bronchoalveolar lavage, transbronchial lung biopsy (T'BLB), and evaluation with special stains (e.g., methenamine silver or Giemsa); laboratory findings, such as complete blood count and blood chemistry; and symptoms, pneumonia severity index (PSI), and radiological findings at the time of $P$. jirovecii PCR testing.

We investigated the clinical outcomes of the two groups regarding the decision to treat (or not to treat) PCP and in-hospital mortality.

We defined improvement as clinical recovery and discharge within 1 month after treatment. Causes of death were divided into four categories: acute respiratory distress syndrome (ARDS), sepsis, multiorgan failure, and 
cardiac arrest. We used the Berlin definition to diagnose ARDS [12]. Sepsis was defined as the presence (probable or documented) of infection together with systemic manifestations of infection [13]. Organ dysfunction was defined according to Marshall et al. [14]. Cardiac arrest was defined as the cessation of cardiac mechanical activity with loss of effective circulation, as determined by the absence of a palpable central pulse [15].

\section{$P$. jirovecii polymerase chain reaction}

A PCR method for the detection of P. jirovecii DNA was developed at our institution. In detail, DNA was extracted from bronchial washing fluid or BALF using a QIAamp mini kit (Qiagen, Hilden, Germany), and the pneumocystis mitochondrial region (mtLSU rRNA) was amplified using nested PCR $[16,17]$. In the two amplification rounds, external and internal primers made by our institute were used, and Taq DNA polymerase (Promega, Southampton, UK) was used throughout the study. Each round comprised 40 cycles of amplification. The amplification products were visualized using ultraviolet light following agarose gel electrophoresis and staining with ethidium bromide. Extreme care was taken to eliminate the possibility of false-positive reactions caused by contamination. All experiments were performed at least twice.

\section{Statistical analysis}

Continuous variables were presented as mean \pm standard error of the mean and analyzed using two independent-sample $t$ tests. Categorical variables were presented as frequencies and percentages, and analyzed using Pearson chi-square test or Fisher exact test for discrete variables. Univariate and multivariate logistic regression analyses were performed to analyze the risk factors for clinical PCP development, with calculation of odds ratios (ORs), 95\% confidence intervals (CIs), and $p$ values. All tests were two sided and $p<0.05$ were considered to be statistically significant. All analyses were performed with the SPSS version 18.o (SPSS Inc., Chicago, IL, USA).

\section{RESULTS}

\section{Baseline demographics of the study population}

P. jirovecii PCR testing of bronchial washing fluid or

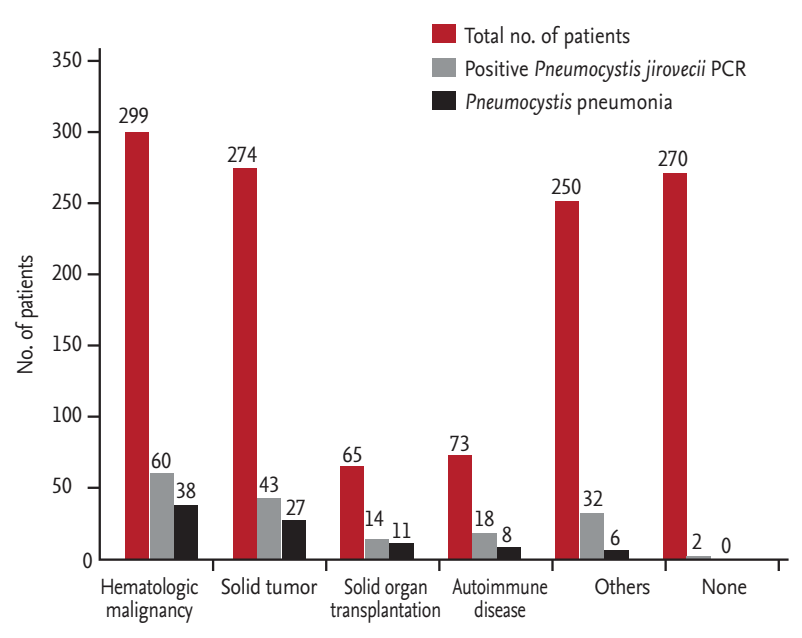

Figure 1. Analysis of underlying diseases among positive Pneumocystis jirovecii polymerase chain reaction (PCR) patients.

BALF yielded positive results for 169 of 1,233 patients (13.7\%). PCP was diagnosed in 90 (53.3\%) of these patients. Among P. jirovecii PCR-positive patients, PCP was present in larger percentages of patients with hematological disease, those who had undergone solid organ transplantation, and those with autoimmune disease (Fig. 1).

Baseline data for the PCP and non-PCP groups are summarized in Table 1. The PCP group was significantly younger than the non-PCP group $(55.3 \pm 1.7$ years vs. $61.1 \pm 1.9$ years, $p=0.02$ ). Compared with the non-PCP group, the PCP group had significantly higher percentages of hematological malignancies ( $42 \%$ vs. $28 \%, p<$ 0.001 ), solid tumors (30\% vs. $20 \%, p<0.001$ ), and solid organ transplant recipients ( $12 \%$ vs. $4 \%, p<0.001)$. No significant difference in previous steroid treatment, use of immunosuppressants (e.g., cyclosporine, tacrolimus), or methotrexate use or chemotherapy was observed between groups.

\section{Comparison of clinical manifestations in PCP and non-PCP patients with $P$. jirovecii PCR positivity}

Laboratory data from the two groups are shown in Table 2. Serum C-reactive protein levels were significantly higher in the PCP group than in the non-PCP group (11.4 $\pm 0.9 \mathrm{mg} / \mathrm{dL}$ vs. $8 \pm 0.8 \mathrm{mg} / \mathrm{dL}, p=0.01)$. Serum albumin levels were significantly lower in the PCP group (2.9 \pm $0.1 \mathrm{~g} / \mathrm{dL}$ vs. $3.2 \pm 0.1 \mathrm{~g} / \mathrm{dL}, p<0.001$ ). No significant difference in the white blood cell count, serum level of lactate 
Table 1. Baseline characteristics

\begin{tabular}{|c|c|c|c|}
\hline Characteristic & $\operatorname{PCP}(n=90)$ & Non-PCP $(n=79)$ & $p$ value \\
\hline Age, yr & $55 \cdot 3 \pm 1.7$ & $61.1 \pm 1.9$ & 0.020 \\
\hline Male sex & $56(62)$ & $52(66)$ & 0.630 \\
\hline Underlying disease & & & $<0.001$ \\
\hline Hematological malignancies & $38(42)$ & $22(28)$ & \\
\hline Solid tumors & $27(30)$ & $16(20)$ & \\
\hline Solid organ transplant recipients & $11(12)$ & $3(4)$ & \\
\hline Autoimmune disease & $8(9)$ & $10(13)$ & \\
\hline Others $^{\mathrm{a}}$ & $5(6)$ & $26(33)$ & \\
\hline None & $1(1)$ & $2(2)$ & \\
\hline Previous steroid treatment & $32(36)$ & $18(23)$ & 0.070 \\
\hline Immunosuppressants $^{\mathrm{b}}$ & $34(38)$ & $20(25)$ & 0.080 \\
\hline Chemotherapy within 1 month & $21(23)$ & $11(14)$ & 0.120 \\
\hline
\end{tabular}

Values are presented as mean \pm SEM or number $(\%)$.

PCP, Pneumocystis pneumonia.

${ }^{a}$ Others include liver cirrhosis, pulmonary tuberculosis, end stage renal disease, interstitial lung disease, and chronic obstructive lung disease.

${ }^{\mathrm{b}}$ Immunosuppressants include cyclosporine, tacrolimus, and methotrexate.

Table 2. Comparison of clinical manifestations between PCP and non-PCP patients with positive Pneumocystis jirovecii polymerase chain reaction

\begin{tabular}{|c|c|c|c|}
\hline Variable & $\operatorname{PCP}(n=90)$ & Non-PCP $(\mathrm{n}=79)$ & $p$ value \\
\hline \multicolumn{4}{|l|}{ Lab data } \\
\hline WBC, $/ \mathrm{mm}^{3}$ & $9,066.4 \pm 641$ & $8,240.1 \pm 693.7$ & 0.380 \\
\hline Lymphocyte, /mm³ & $1,352.6 \pm 405.7$ & $1,180.1 \pm 118.8$ & 0.740 \\
\hline $\mathrm{ANC}, / \mathrm{mm}^{3}$ & $7,224.9 \pm 521.4$ & $6,124 \cdot 1 \pm 562.3$ & 0.150 \\
\hline $\mathrm{LDH}, \mathrm{U} / \mathrm{L}$ & $1,027 \cdot 3 \pm 165 \cdot 5$ & $663.7 \pm 53.9$ & 0.050 \\
\hline $\mathrm{CRP}, \mathrm{mg} / \mathrm{dL}$ & $11.4 \pm 0.9$ & $8 \pm 0.8$ & 0.010 \\
\hline Albumin, g/dL & $2.9 \pm 0.1$ & $3.2 \pm 0.1$ & $<0.001$ \\
\hline $\mathrm{PaO}_{2}, \mathrm{mmHg}$ & $76.8 \pm 2.2$ & $76.5 \pm 2.8$ & 0.940 \\
\hline \multicolumn{4}{|l|}{ Symptoms } \\
\hline Fever & $57(63)$ & $40(51)$ & 0.100 \\
\hline Cough & $33(37)$ & $31(39)$ & 0.730 \\
\hline Sputum & $30(33)$ & $28(35)$ & 0.770 \\
\hline Dyspnea & $56(62)$ & $24(30)$ & $<0.001$ \\
\hline Pneumonia severity index & $102.1 \pm 3.4$ & $96.6 \pm 4$ & 0.300 \\
\hline \multicolumn{4}{|l|}{ Radiology } \\
\hline Bilateral lung GGO & $72(80)$ & $50(63)$ & 0.020 \\
\hline
\end{tabular}

Values are presented as mean \pm SEM or number $(\%)$.

PCP, Pneumocystis pneumonia; WBC, white blood cell; ANC, absolute neutrophil count; LDH, lactate dehydrogenase; CRP, C-reactive protein; $\mathrm{PaO}_{2}$, partial pressure of arterial oxygen; GGO, ground glass opacity. 
dehydrogenase, $\mathrm{PaO}_{2}$, or PSI was observed between the groups. Regarding symptoms, the incidence of dyspnea was significantly higher in the PCP group than in the non-PCP group ( $62 \%$ vs. $30 \%, p<0.001$ ). Radiological findings revealed a higher incidence of ground-glass opacities in the bilateral lungs in the PCP group $(80 \%$ vs. $63 \%, p=0.02$ ).

\section{Comparison of clinical outcomes in PCP and non- PCP patients with $P$. jirovecii $P C R$ positivity}

Clinical outcomes of the two groups are shown in Table 3. Fifty-six patients $(62 \%)$ in the PCP group improved with TMP-SMX treatment, and 66 of 79 patients (84\%) in the non-PCP group recovered in their clinical course due to other following treatments; supportive care (6/66), antibiotics (48/66), antituberculosis medication (3/66), antifungal (1/66), others (steroid, tamiflu, etc.; 8/66).

The fatality rate was higher in the PCP group than in the non-PCP group (38\% vs. $16 \%, p=0.002$ ). In the PCP group $(\mathrm{n}=90), 34$ patients died of ARDS, sepsis, or multiorgan failure. Among them, two patients died due to exacerbation of PCP despite treatment.

In the non-PCP group, 66 patients improved following various treatments: supportive care (6/66), antibiotics (48/66), antituberculosis medication (3/66), antifungals $(1 / 66)$, and others (e.g., steroids, tamiflu; 8/66). No patient in this group died due to PCP worsening.

\section{Predictive parameters for PCP among $P$. jirovecii PCR-positive patients}

In Table 4, multivariate logistic regression analysis revealed that the independent risk factors for PCP diagnosis in P. jirovecii PCR-positive patients were underlying conditions, such as hematological malignancies, solid tumors, and solid organ transplant receipt (OR, 8.61; 95\% CI, 3.08 to 24.09 ; $p<0.001$ ); dyspnea (OR, 4.59; $95 \%$ CI, 1.95 to $10.76 ; p<0.001)$; age > 60 years (OR, 1.03; $95 \%$ CI, 1.0 to 1.06; $p=0.02)$; and albumin < 2.9 g/dL (OR, 3.08; $95 \%$ CI, 1.34 to $7.08 ; p=0.01$ ).

\section{DISCUSSION}

In the current study, we divided the P.jirovecii PCR-positive population into two groups-patients with clinical diagnoses of PCP who received TMP-SMX treatment, and those diagnosed simply with colonization-and compared the two groups to evaluate the risk factors for PCP. The results showed that PCP was more common in P.jirovecii PCR-positive patients aged $<60$ years; those with hematological malignancies and solid tumors; those who were solid organ transplant recipients; those with low albumin levels; and those with symptoms of dyspnea. Moreover, colonization was detected in a considerable percentage of $P$. jirovecii PCR-positive immunocompromised patients, indicating that they did not require treatment.

Table 3. Clinical outcome between PCP and non-PCP patients with positive Pneumocystis jirovecii polymerase chain reaction

\begin{tabular}{|c|c|c|c|}
\hline Variable & $\operatorname{PCP}(n=90)$ & Non-PCP $(\mathrm{n}=79)$ & $p$ value \\
\hline Improved & $56(62)$ & $66^{\mathrm{a}}(84)$ & 0.002 \\
\hline Death & $34(38)$ & $13(16)$ & \\
\hline \multicolumn{4}{|l|}{ Cause of death } \\
\hline ARDS & $12 / 34$ & $3 / 13$ & \\
\hline Sepsis & $5 / 34$ & $2 / 13$ & \\
\hline Multiorgan failure & $12 / 34$ & $5 / 13$ & \\
\hline Cardiac arrest & $3 / 34$ & $3 / 13$ & \\
\hline Death related to $\mathrm{PCP}$ & $2(2)$ & o & \\
\hline
\end{tabular}

Values are presented as number (\%).

PCP, Pneumocystis pneumonia; ARDS, acute respiratory distress syndrome.

${ }^{a}$ Improvement in the non-PCP group $(\mathrm{n}=66)$ is due to the following; supportive care $(6 / 66)$, antibiotics (48/66), anti-tuberculosis medication (3/66), anti-fungal (1/66), others (steroid, tamiflu, etc.; 8/66). 
Table 4. Multivariate regression analysis for the risk factor of Pneumocystis pneumonia development among positive Pneumocystis jirovecii polymerase chain reaction patients

\begin{tabular}{lccc}
\hline Variable & OR & $95 \%$ CI & $p$ value \\
\hline Hematologic malignancies, solid tumors, or SOT & 8.61 & $3.08-24.09$ & $<0.001$ \\
Dyspnea & 4.59 & $1.95-10.76$ & $<0.001$ \\
Age $<60$ yr & 1.03 & $1.00-1.06$ & 0.020 \\
Albumin $<2.9 \mathrm{~g} / \mathrm{dL}$ & 3.08 & $1.34-7.08$ & 0.010 \\
\hline
\end{tabular}

OR, odds ratio; CI, confidence interval; SOT, solid organ transplantation.

The diagnosis of PCP in clinical practice is challenging. First, the diagnostic yields of direct staining and lung biopsy are low, ranging from $38 \%$ to $53 \%[6,7]$; values are lower for immunocompromised patients without HIV than for patients with HIV. Second, some methods, such as TBLB, are invasive and often inaccessible in a clinical setting. Lastly, the symptoms and radiological findings at the time of PCP development are frequently nonspecific and ambiguous. Recently, the $P$. jirovecii PCR method has helped to improve the diagnostic sensitivity of PCP diagnosis from BALF and induced sputum. However, the differentiation of infection and colonization in the PCR-based diagnosis of PCP can be difficult, particularly in patients without HIV [18]. The extent of colonization has been debated, with different rates of $P$. jirovecii carriage reported. Nevez et al. [19] reported that $20 \%$ of $169 \mathrm{HIV}$-negative patients with moderate or severe immunosuppression showed $P$. jirovecii positivity according to nested PCR; none of these patients developed PCP. In the current study, 37\% of patients with hematological malignancies, $37 \%$ of those with solid tumors, $21 \%$ of solid organ transplant recipients, $56 \%$ of patients with autoimmune diseases, and $81 \%$ of patients with other diseases had P. jirovecii colonization. Most $(83.5 \%, \mathrm{n}=66)$ patients in the non-PCP group, who were $P$. jirovecii PCR-positive according to analysis of bronchoscopic specimens, recovered without treatment of PCP, indicating that they were colonized or had subclinical infection. This finding suggests that a fair number of patients with P.jirovecii PCR positivity do not need to be treated, despite their low immune status. On the other hand, P. jirovecii colonization has several clinical implications. It may increase the risk of acute PCP in susceptible hosts, or result in the transmission of $P$. jirovecii to others $[16,20]$. Nevertheless, the duration of colonization before the development of PCP and the efficacy of treating colonization to prevent disease are unknown [21].

In our study, we attempted to determine the predictive factors of PCP among P. jirovecii-positive patients by comparing the PCP and non-PCP groups. The PCP group contained significantly larger percentages of patients with hematological malignancies, those with solid tumors, and solid organ transplant recipients among various underlying immunocompromising diseases. The higher incidence of PCP among these patients might be due primarily to a higher net state of immunosuppression caused by the underlying disease and the intensity of chemotherapy or immunosuppressant use in these patients [22].

Intriguingly, the current study showed that patients in the PCP group were significantly younger than those in the non-PCP group. Previous reports showed an association between age $>60$ years and pulmonary Pneumocystis colonization, especially in patients with rheumatoid arthritis [23,24]. Advanced age itself may compromise the immune system, leads to increased Pneumocystis colonization. This hypothesis should be tested in future studies.

Dyspnea symptoms were significantly more common in the PCP group than in the non-PCP group. However, interestingly, $\mathrm{PaO}_{2}$ did not differ between groups. $P$. jirovecii PCR-positive patients with subjective symptoms of dyspnea despite no evidence of hypoxia should be evaluated carefully for evidence of PCP. Significant hypoalbuminemia, suggesting compromised nutritional status, was noted in the PCP group compared with the non-PCP group. Hypoalbuminemia has been reported as a poor prognostic factor for PCP [25].

Regarding mortality, whereas 34 patients (38\%) in the PCP group died despite treatment, 66 patients (84\%) in the non-PCP group improved without treatment of 
PCP. In the clinical PCP group, the main causes of death were ARDS, multiorgan failure, sepsis, and cardiac arrest. Some patients may have died due to conditions other than PCP, whereas others may have died due to refractoriness to PCP therapy. In contrast, in the nonPCP group, most patients survived with adequate treatment other than PCP therapy. This finding suggests a considerable incidence of colonization in patients with P. jirovecii PCR positivity, despite their significant immunodeficient status.

The limitations of this study were its retrospective nature and the use of a clinical diagnosis as the gold standard for PCP, as opposed to the performance of lung biopsy in all patients. However, lung biopsy is quite invasive and often extremely risky in these patients. In addition, some studies have failed to show benefits in terms of PCP diagnosis [26].

Another limitation was that we used conventional PCR methods instead of quantitative real-time PCR assays, which have been reported to be more reliable for the diagnosis of PCP [27,28]. Quantitative real-time PCR assays, along with the use of serum markers such as B-D glucan, enable differentiation between PCP and Pneumocystis colonization [27-30]. However, these assays are costly and their cut-off values are not standardized; therefore, their application in clinical practice remains limited.

\section{KEY MESSAGE}

1. Not all Pneumocystis jirovecii polymerase chain reaction (PCR)-positive patients need to be treated for Pneumocystis pneumonia (PCP), considering their low immune status.

2. In cases of $P$. jirovecii PCR positivity, the risk of definitive PCP is higher in patients aged $<60$ years, those with hematological malignancies or solid tumors, solid organ transplant recipients, patients with low albumin levels $(<2.9 \mathrm{~g} / \mathrm{dL})$, and those with symptoms of dyspnea.

3. Treatment of PCP should be considered in these patients.

\section{Conflict of interest}

No potential conflict of interest relevant to this article was reported.

\section{REFERENCES}

1. Thomas CF Jr, Limper AH. Pneumocystis pneumonia. N Engl J Med 2004;350:2487-2498.

2. Overgaard UM, Helweg-Larsen J. Pneumocystis jiroveci pneumonia (PCP) in HIV-1-negative patients: a retrospective study 2002-2004. Scand J Infect Dis 2007;39:589-595.

3. Roblot F, Godet C, Le Moal G, et al. Analysis of underlying diseases and prognosis factors associated with Pneumocystis carinii pneumonia in immunocompromised HIV-negative patients. Eur J Clin Microbiol Infect Dis 2002;21:523-531.

4. Reid AB, Chen SC, Worth LJ. Pneumocystis jirovecii pneumonia in non-HIV-infected patients: new risks and diagnostic tools. Curr Opin Infect Dis 2011;24:534-544.

5. Morris A, Wei K, Afshar K, Huang L. Epidemiology and clinical significance of pneumocystis colonization. J Infect Dis 2008;197:10-17.

6. Leibovitz E, Pollack H, Moore T, et al. Comparison of PCR and standard cytological staining for detection of Pneumocystis carinii from respiratory specimens from patients with or at high risk for infection by human immunodeficiency virus. J Clin Microbiol 1995;33:3004-3007.

7. Lipschik GY, Gill VJ, Lundgren JD, et al. Improved diagnosis of Pneumocystis carinii infection by polymerase chain reaction on induced sputum and blood. Lancet 1992;340:203-206.

8. Mekinian A, Durand-Joly I, Hatron PY, et al. Pneumocystis jirovecii colonization in patients with systemic autoimmune diseases: prevalence, risk factors of colonization and outcome. Rheumatology (Oxford) 2011;50:569-577.

9. Wakefield AE, Pixley FJ, Banerji S, et al. Detection of Pneumocystis carinii with DNA amplification. Lancet 1990;336:451-453.

10. Kovacs JA, Masur H. Evolving health effects of Pneumocystis: one hundred years of progress in diagnosis and treatment. JAMA 2009;301:2578-2585.

11. The National Institutes of Health-University of California Expert Panel for Corticosteroids as Adjunctive Therapy for Pneumocystis Pneumonia. Consensus statement on the use of corticosteroids as adjunctive therapy for pneumocystis pneumonia in the acquired immunodeficiency syndrome. N Engl J Med 1990;323:1500-1504.

12. ARDS Definition Task Force, Ranieri VM, Rubenfeld GD, et al. Acute respiratory distress syndrome: the Berlin Definition. JAMA 2012;307:2526-2533. 
13. Levy MM, Fink MP, Marshall JC, et al. 2001 SCCM/ESICM/ACCP/ATS/SIS International Sepsis Definitions Conference. Crit Care Med 2003;31:1250-1256.

14. Marshall JC, Cook DJ, Christou NV, Bernard GR, Sprung CL, Sibbald WJ. Multiple organ dysfunction score: a reliable descriptor of a complex clinical outcome. Crit Care Med 1995;23:1638-1652.

15. Fishman GI, Chugh SS, Dimarco JP, et al. Sudden cardiac death prediction and prevention: report from a National Heart, Lung, and Blood Institute and Heart Rhythm Society Workshop. Circulation 2010;122:2335-2348.

16. Miller RF, Ambrose HE, Wakefield AE. Pneumocystis carinii f. sp. hominis DNA in immunocompetent health care workers in contact with patients with P. carinii pneumonia. J Clin Microbiol 2001;39:3877-3882.

17. Tsolaki AG, Beckers P, Wakefield AE. Pre-AIDS era isolates of Pneumocystis carinii f. sp. hominis: high genotype similarity with contemporary isolates. J Clin Microbiol 1998;36:90-93.

18. Tasaka S, Tokuda H. Pneumocystis jirovecii pneumonia in non-HIV-infected patients in the era of novel immunosuppressive therapies. J Infect Chemother 2012;18:793806.

19. Nevez G, Jounieaux V, Linas MD, et al. High frequency of Pneumocystis carinii sp.f. hominis colonization in HIV-negative patients. J Eukaryot Microbiol 1997;44:36S.

20. Chabe M, Dei-Cas E, Creusy C, et al. Immunocompetent hosts as a reservoir of pneumocystis organisms: histological and rt-PCR data demonstrate active replication. Eur J Clin Microbiol Infect Dis 2004;23:89-97.

21. Morris A, Norris KA. Colonization by Pneumocystis jirovecii and its role in disease. Clin Microbiol Rev 2012;25:297-317.

22. Torres HA, Chemaly RF, Storey R, et al. Influence of type of cancer and hematopoietic stem cell transplantation on clinical presentation of Pneumocystis jiroveci pneumonia in cancer patients. Eur J Clin Microbiol Infect Dis 2006;25:382-388.
23. Fritzsche C, Riebold D, Munk-Hartig A, Klammt S, Neeck G, Reisinger E. High prevalence of Pneumocystis jirovecii colonization among patients with autoimmune inflammatory diseases and corticosteroid therapy. Scand J Rheumatol 2012;41:208-213.

24. Mori S, Cho I, Ichiyasu H, Sugimoto M. Asymptomatic carriage of Pneumocystis jiroveci in elderly patients with rheumatoid arthritis in Japan: a possible association between colonization and development of Pneumocystis jiroveci pneumonia during low-dose MTX therapy. Mod Rheumatol 2008;18:240-246.

25. Kovacs JA, Hiemenz JW, Macher AM, et al. Pneumocystis carinii pneumonia: a comparison between patients with the acquired immunodeficiency syndrome and patients with other immunodeficiencies. Ann Intern Med 1984;100:663-671.

26. White DA, Wong PW, Downey R. The utility of open lung biopsy in patients with hematologic malignancies. Am J Respir Crit Care Med 2000;161(3 Pt 1):723-729.

27. Fujisawa T, Suda T, Matsuda H, et al. Real-time PCR is more specific than conventional PCR for induced sputum diagnosis of Pneumocystis pneumonia in immunocompromised patients without HIV infection. Respirology 2009;14:203-209.

28. Muhlethaler K, Bogli-Stuber K, Wasmer S, et al. Quantitative PCR to diagnose Pneumocystis pneumonia in immunocompromised non-HIV patients. Eur Respir J 2012;39:971-978.

29. Matsumura $Y$, Ito $Y$, Iinuma $Y$, et al. Quantitative real-time PCR and the $(1 \rightarrow 3)-\beta$-D-glucan assay for differentiation between Pneumocystis jirovecii pneumonia and colonization. Clin Microbiol Infect 2012;18:591-597.

30. Tasaka S, Kobayashi S, Yagi K, et al. Serum $(1 \rightarrow 3)$ $\beta$-D-glucan assay for discrimination between Pneumocystis jirovecii pneumonia and colonization. J Infect Chemother 2014;20:678-681. 